\title{
An Eight-Week Trial Investigating the Efficacy and Tolerability of Atorvastatin for Children and Adolescents With Heterozygous Familial Hypercholesterolemia
}

\author{
Kuan Gandelman · Paul Glue $\cdot$ Rachel Laskey $\cdot$ \\ Jonathan Jones $\cdot$ Robert LaBadie $\cdot$ Leiv Ose
}

Received: 20 August 2010/ Accepted: 3 January 2011/Published online: 23 January 2011

(C) The Author(s) 2011. This article is published with open access at Springerlink.com

\begin{abstract}
This study aimed to assess the efficacy and tolerability of atorvastatin in Tanner stage (TS) 1 patients ages 6 to 10 years and TS $\geq 2$ patients ages 10 to $<18$ years with genetically confirmed heterozygous familial hypercholesterolemia $(\mathrm{HeFH})$ and a low density lipoprotein cholesterol (LDL-C) level of $4 \mathrm{mmol} / \mathrm{l}(155 \mathrm{mg} / \mathrm{dl})$ or higher. In this open-label, 8-week study, 15 TS 1 children were treated initially with atorvastatin $5 \mathrm{mg} /$ day and $24 \mathrm{TS}$ $\geq 2$ children with $10 \mathrm{mg} / \mathrm{day}$. Doses were doubled at week 4 if the LDL-C target $(<3.35 \mathrm{mmol} / \mathrm{l}[130 \mathrm{mg} / \mathrm{dl}])$ was not achieved. The efficacy variables were the percentage change from baseline in LDL-C, total cholesterol (TC), triglycerides (TG), high density lipoprotein cholesterol (HDL-C), very low density lipoprotein cholesterol (VLDLC), and apolipoprotein (Apo) A-I and Apo B. Safety evaluations included clinical monitoring, subject-reported adverse events (AEs), vital signs, and clinical laboratory tests. The mean values for LDL-C, TC, VLDL-C, and Apo B decreased by week 2 among all TS 1 and TS $\geq 2$ patients, whereas TG, HDL-C, and Apo A-I varied considerably from week to week. After 8 weeks, the mean reduction in
\end{abstract}

K. Gandelman $(\bowtie) \cdot$ R. Laskey $\cdot$ R. LaBadie

Pfizer Inc, 235 East 42nd Street, New York, NY 10017, USA

e-mail: kuan.gandelman@pfizer.com

P. Glue

Dunedin School of Medicine, P.O. Box 913, Dunedin,

New Zealand

J. Jones

Pfizer Ltd, Walton Oaks, Surrey, UK

L. Ose

Department of Medicine, Oslo University Hospital,

Rikshospitalet, Oslo, Norway
LDL-C was $-40.7 \% \pm 8.4$ for the TS 1 children and $-39.7 \% \pm 10.3$ for the TS $\geq 2$ children. For the TS 1 patients, the mean reductions were $-34.1 \% \pm 6.9$ for TC and $-6.0 \% \pm 32.1$ for TG. The corresponding changes for the TS $\geq 2$ patients were $-35.6 \% \pm 9.5$ for TC and $-21.1 \% \pm 29.7$ for TG. Four patients experienced mild to moderate treatment-related AEs. No serious AEs or discontinuations were reported. Overall, no difference in safety or tolerability was observed between the younger and older cohorts. Across the range of exposures after atorvastatin 5 to $10 \mathrm{mg}$ (TS 1) or atorvastatin 10 to $20 \mathrm{mg}$ (TS $\geq 2$ ) doses for 8 weeks, clinically meaningful reductions in LDL-C, TC, VLDL-C, and Apo were observed with atorvastatin in pediatric patients who had HeFH. Atorvastatin also was well tolerated in this population.

Keywords Heterozygous familial hypocholesterolemia . Low density lipoprotein cholesterol $\cdot$ Statin

Familial hypercholesterolemia $(\mathrm{FH})$ is a common inherited autosomal dominant disorder that causes markedly elevated low density lipoprotein cholesterol (LDL-C) levels, resulting in increased morbidity of coronary heart disease, with premature death [13]. The severity of atherosclerosis is correlated with the duration of hypercholesterolemia, and early initiation of statin therapy for children with $\mathrm{FH}$ was shown to be beneficial in preventing atherosclerosis [18].

Several well-controlled studies have demonstrated that the short-term safety and effectiveness of the 3-hydroxy-3methylglutaryl coenzyme A reductase (HMG-CoA) inhibitors, or statins, in children and adolescents have been similar to those observed in adults $[1,7,14,16,19,21]$. Recently, both the American Heart Association (AHA) [17] and the American Academy of Pediatrics (AAP) [4] 
have recommended that target levels for LDL-C as low as less than $130 \mathrm{mg} / \mathrm{dl}(3.35 \mathrm{mmol} / \mathrm{l})$ should be set for children who are heterozygous for $\mathrm{FH}$, and that statins, among other classes of drugs, should be considered as a first-line agent. In addition, AHA recommends that after a trial of dietary changes, drug therapy should ideally be started at Tanner stage (TS) 2 or higher.

However, for children and adolescents with high-risk lipid abnormalities who have additional risk factors or high-risk conditions, in selected cases, clinicians may consider initiation for children younger than 10 years. The AAP has recommended that statins be started as early as 8 years of age $[6,14,21]$ based on a few studies that examined the efficacy and safety of statins in prepubertal children and children 8 to 10 years of age.

Currently, pravastatin is the only drug approved for children with heterozygous $\mathrm{FH}(\mathrm{HeFH})$ as young as 8 years of age, whereas other available statins are approved for children older than 10 years. To date, available study data are limited with regard to initiation of pharmacologic treatment for TS 1 children, to what target LDL-C levels should be reached for FH or other high risk children, and to the long term effects of statins on growth and sexual development. This may be due in part to the lack of availability of an appropriate pediatric formulation for the treatment of patients at younger ages. The development of an appropriate pediatric formulation has been identified as a current unmet pediatric need in cardiovascular products by the Pediatric Working Party, a view also adopted and published by the CHMP in November 2006 [8].

A chewable tablet formulation was developed as an alternative treatment option, together with availability of a lower-strength dose $(5 \mathrm{mg})$ for use with younger pediatric patients, as part of a Pediatric Investigation Plan recommended in the Written Request by the EMEA Paediatric Committee (PDCO) in 2009 [10]. In keeping with the need to obtain appropriate data yet limit the exposure of younger children, this 8-week pharmacokinetic and pharmacodynamic (PK-PD) study used a population-based approach recommended and endorsed by the Pediatric Committee.

The current study aimed to evaluate the short-term efficacy and tolerability of atorvastatin for $\mathrm{HeFH}$ children. The patient cohort included a subgroup of TS 1 patients, younger than those enrolled in most previous pediatric studies, treated for 8-weeks with a chewable tablet developed for pediatric use. The chewable tablet formulation was previously shown to have bioequivalence to the marketed tablet formulation. Other objectives of this PK-PD study including a description of the pharmacokinetics of atorvastatin in children ages 6 to 17 years, characterization of the relationship between atorvastatin and metabolite exposure (pharmacokinetics), changes in lipid parameters (pharmacodynamics), and possible recommendation of a safe and efficacious lower dose $(<10 \mathrm{mg}$ ) for children ages 6 to 10 years, are described in a separate publication.

\section{Methods}

\section{Study Design}

From December 2008 to May 2009, 39 children and adolescents with genetically confirmed HeFH and an LDL-C of $4 \mathrm{mmol} / \mathrm{l}(\geq 155 \mathrm{mg} / \mathrm{dl})$ or more were enrolled in this 8-week, open-label, uncontrolled study (NCT00739999). The study participants were stratified into two cohorts according to their Tanner stage, determined at the screening visit.

The study was conducted at three centers in Greece, Norway, and Canada in compliance with the ethical principles originating in or derived from the Declaration of Helsinki and in compliance with all International Conference on Harmonization (ICH) Good Clinical Practice (GCP) Guidelines. In addition, all local regulatory requirements were followed, particularly those affording greater protection of the study participants' safety. Written informed consent was obtained from each child's parent or legal guardian, and the minor's assent also was required when it could be obtained.

\section{Patients}

Eligible patients included girls and boys ages 6 to $<18$ years. Children and adolescents 10 to 17 years of age were to be at TS $\geq 2$ in their development, whereas children 6 to 10 years of age were confirmed to be at TS 1 . All patients had genetically verified $\mathrm{HeFH}$, as evidenced by LDL-C receptor mutation analysis [20] or by the lymphocyte test [3]. If subjects tested negative for the most common LDL receptor mutations, detection of FH was to be carried out by assaying LDL-C receptors on lymphocytes. In addition, all patients must have had a plasma LDL-C level of $4 \mathrm{mmol} / \mathrm{l}(155 \mathrm{mg} / \mathrm{dl})$ or higher at the screening visit. The patients, otherwise in good health based on the results of routine clinical laboratory tests, physical examinations, and normal electrocardiograms (ECGs) performed at screening, were judged by the investigator to be good candidates for the study.

Subjects previously receiving probucol were required to have stopped the treatment for 6 months or more to be eligible. If a subject had been receiving statins previously, a requisite washout period 6 weeks or longer was required before entry to the study. Patients previously receiving other antihyperlipidemic therapies were considered for screening after a washout period of 4 weeks or longer before entering this study. However, due to the competitive 
enrollment between the three study sites, most, if not all, the subjects enrolled in this study had not been previously treated for dyslipidemia and therefore did not require a washout period. Use of other prescription or over-thecounter drugs and dietary supplements were stopped 7 days or 5 half-lives (whichever was longer) before the first dose of study medication.

\section{Treatment}

Eligible patients entered the treatment phase of the study 1 to 4 weeks after the screening visit and received daily atorvastatin for 8 weeks. The starting dose of atorvastatin was $10 \mathrm{mg} /$ day (formulated as a solid tablet) for the subjects 10 to 17 years of age (i.e., $\mathrm{TS} \geq 2$ ) and $5 \mathrm{mg} /$ day (formulated as a chewable tablet for pediatric use) for the subjects 6 to 10 years of age (i.e., TS 1). If a subject had not attained the target plasma LDL-C level $(<3.35 \mathrm{mmol} / \mathrm{l}$ $[<130 \mathrm{mg} / \mathrm{dl}])$ at week 4 , the atorvastatin dose could be doubled. Compliance (defined as $80-120 \%$ pill usage) was assessed by examination of completed dosing diaries and by tablet counts. Diets were not monitored during the study period.

\section{Efficacy Assessments}

The primary objective was to evaluate the effects of atorvastatin on plasma lipid levels after 8 weeks of treatment. The efficacy parameters were the mean percentage changes from baseline for LDL-C, total cholesterol (TC), triglycerides (TG), high density lipoprotein cholesterol (HDL-C), very low density lipoprotein-cholesterol (VLDL-C), and apolipoprotein (Apo) A-I, and Apo B. Because the patients could have doubled their dose at week 4, additional information is provided regarding those patients.

Fasting (at least $10 \mathrm{~h}$ from the last meal) blood samples ( $2 \mathrm{ml}$ ) were taken for lipid profiling at screening (visit 1), week 2 (visit 3), week 4 (visit 4), week 6 (visit 5), and week 8 (visit 6). Fasting was optional at weeks 2 and 6 because clinic visits were in the afternoon. Lipid values obtained at screening were considered as baseline values to conserve the amount of blood drawn at week 1 (visit 2).

An optional assessment in the current study, flowmediated dilation (FMD), was conducted at two of the centers that had FMD measurement facilities available. These measurements were designed to provide exploratory information about endothelial function in the brachial arteries via high-resolution ultrasound evaluation of arterial diameter responses to increased blood flow [2]. The subjects were not required to participate in the FMD analysis.

The ultrasound machine was equipped with a 5- to $14-\mathrm{MHz}$ vascular (near field) transducer and vascular imaging software. The ultrasound images of the brachial artery were obtained during the diastolic phase of the brachial artery (identified by R-wave of the electrocardiogram [ECG] or the smallest brachial artery at rest via visual inspection). The sequential images of the brachial artery enabled reconstruction of the brachial arterial lumen diameter over the entire scan. Experienced sonographers followed a standardized methodology, and scans were batch analyzed at the individual FMD sites and blinded for time point to minimize observer bias.

The FMD was assessed at weeks 0 and 8 . For each scan, baseline vessel size in millimeters, peak vessel size in millimeters, and FMD as a percentage were entered into the FMD database. From these parameters, FMD was calculated as

$$
\begin{aligned}
\text { Brachial FMD }= & {[(\text { maximum diameter }} \\
& - \text { baseline diameter }) / \text { baseline diameter }] \\
& \times 100 \%
\end{aligned}
$$

\section{Safety Assessments}

Safety and tolerability were evaluated throughout the study by review of patient reports, investigator observations, and results of specific tests and measurements. The study investigators assessed all adverse events (AEs) for level of intensity and relationship to the study drug.

Routine clinical laboratory evaluations comprised hematology and biochemistry tests performed at baseline; at weeks 2, 4, and 6; and at the final visit at week 8 . Urinalysis, physical examination (including height, weight, and TS), and 12-lead ECG were performed at screening and at week 8 . Blood pressure and pulse rate were checked at screening, week 4 , and week 8 . The clinical laboratory sample analyses were performed by Quintiles Laboratories in the United States and the United Kingdom.

Any safety laboratory values exceeding predefined thresholds were identified and tabulated. All such changes considered clinically significant were to be recorded as AEs. If at any time during the study, a patient's alanine aminotransferase (ALT) or aspartate aminotransferase (AST) plasma levels increased to more than three times the upper limits of normal (ULN), the patient was to be scheduled for a repeat laboratory measurement within 1 week ( \pm 3 days). If the repeat value still exceeded $3 \times$ ULN, the patient's medication was to be reduced by $50 \%$ and a repeat laboratory measurement completed after 2 weeks of therapy with the reduced dose. If the repeat value still exceeded $3 \times \mathrm{ULN}$, the patient was to be withdrawn from the study, and a final physical examination was to be performed. This persistent elevation in ALT or AST was to be reported as a clinically important AE. A patient was discontinued immediately from the study if the creatine phosphokinase (CPK) level was elevated to 
$10 \times$ ULN accompanied by muscle pain, tenderness, or weakness. A persistent plasma CPK elevation of $10 \times \mathrm{ULN}$ or more without muscle pain, tenderness, or weakness also was designated as a prespecified reason for study drug discontinuation.

\section{Statistical Analysis}

The analysis population was defined as all the enrolled subjects who received one or more doses of the study drug and had one or more pharmacodynamic parameter measurements. Change and percentage change from baseline in the lipid parameters were summarized using descriptive statistics by cohort over time.

\section{Results}

Patient Accounting and Demographics

Of the 45 individuals screened in this study, 39 pediatric patients with $\mathrm{HeFH}$ were treated, comprising 15 children at TS 1 and 24 adolescents at TS $\geq 2$. All the patients completed the study. Table 1 shows that all the patients in both

Table 1 Baseline patient demographics

\begin{tabular}{lll}
\hline Parameter & $\begin{array}{l}\text { Tanner stage } 1 \\
(n=15)\end{array}$ & $\begin{array}{l}\text { Tanner stage } \geq 2 \\
(n=24)\end{array}$ \\
\hline Age (years) & & 0 \\
$6-8$ & 7 & 3 \\
$9-10$ & 6 & 14 \\
$11-14$ & $2^{\mathrm{a}}$ & 7 \\
15-17 & 0 & $13.5 \pm 1.9$ \\
Mean (SD), years & $8.7 \pm 1.8$ & $10-17$ \\
Range & $6-12$ & $12(50)$ \\
Male: $n(\%)$ & $8(53)$ & $24(100)$ \\
Race: $n(\%)$ & & \\
White & $15(100)$ & $54.3 \pm 14.7$ \\
Weight $(\mathrm{kg})$ & & $32.0-99.4^{\mathrm{b}}$ \\
Mean $(\mathrm{SD})$ & $32.6 \pm 8.7$ & $160.1 \pm 10.2$ \\
Range & $25.0-54.2$ & $(137.5-182.0)$ \\
Height $(\mathrm{cm})$ & & $20.9 \pm 4.2$ \\
Mean (SD) & $137.4 \pm 9.0$ & $(15.8-32.5)$ \\
Range & $(119.0-151.0)$ & \\
BMI (kg/m $\left.{ }^{2}\right)$ & $17.1 \pm 3.0$ & \\
Mean $(\mathrm{SD})$ & $(13.2-24.7)$ & \\
Range & & \\
\hline BI & &
\end{tabular}

$B M I$ body mass index

a These patients were captured as protocol deviations

b One male weighed $99.4 \mathrm{~kg}$, which was above the 95th percentile for his age. He was included in the study and captured as a protocol deviation cohorts were white and that the proportions of males and females in the two cohorts were similar. Two TS 1 patients were older than 10 years and therefore noted as protocol deviations. Evaluation by a pediatric endocrinologist determined that neither patient had abnormalities in any system.

The patients were treated for a median of 55 days (range, 46-77 days) in the TS 1 cohort and 61.5 days (range, 40-77 days) in the TS $\geq 2$ cohort. The atorvastatin dose levels were doubled after the week 4 visit for more than half of the patients (10 of 15 TS 1 patients and 15 of $24 \mathrm{TS} \geq 2$ patients). Adherence to the study drug regimen was good, with only four patients having a compliance rate lower than $80 \%$ (range, 73-80\%). A total of 16 subjects (2 subjects of TS 1 and 14 subjects of TS 2) from one study center in Canada had visit schedules outside the 7-day range specified by the protocol due to various family or study center scheduling-related reasons.

\section{Efficacy}

Table 2 presents information for all the lipid parameters measured by the Tanner stage cohort and within those cohorts by final dose at week 8 . The baseline mean LDL-C value was $5.87 \mathrm{mmol} / \mathrm{l}$ for the TS1 cohort and $5.81 \mathrm{mmol} / \mathrm{l}$ for the $\mathrm{TS} \geq 2$ cohorts. Administration of daily atorvastatin for 8 weeks resulted in reduced LDL-C, TC, and TG in TS 1 and TS $\geq 2$ children (Table 2 ). Across both cohorts, the mean plasma levels of LDL-C fell from baseline to week 8 by approximately $40 \%$, and TC fell by approximately $35 \%$.

The mean reductions in LDL-C and TC were comparable across both cohorts at week 8 . The baseline TG levels all were within the normal range, with $\mathrm{TS} \geq 2$ patient levels slightly higher than TS 1 patient levels (1.14 vs. $0.89 \mathrm{mmol} /$ 1). Similarly, a larger reduction in the mean plasma TG level was observed in the TS $\geq 2$ patients than in the TS 1 patients $(-21.05 \%$ vs. $-6.02 \%)$. The baseline HDL levels were within the normal range, with the $\mathrm{TS} \geq 2$ patients having slightly lower values than the TS 1 cohort (1.18 vs. $1.35 \mathrm{mmol} / \mathrm{l})$. In both cohorts, nonsignificant reductions from baseline in plasma HDL-C levels were observed, driven by changes in HDL-C among patients whose atorvastatin dose was doubled at week 4.

Over the 8-week study period, LDL-C/HDL-C ratios decreased across both cohorts (data on file). In both cohorts at study end, treatment with atorvastatin also had produced substantial reductions in the mean plasma levels of VLDL$\mathrm{C}$, LDL-C/HDL-C, and Apo B, as well as modest reductions in Apo A-I. The VLDL-C reductions appeared to be greater in the TS $\geq 2$ cohort than in the TS 1 cohort $(-52.84 \%$ vs. $-37.63 \%)$.

Table 2 also shows that patients who did not achieve the recommended treatment goal at week 4 (those whose dose 
Table 2 Mean (SD) percentage changes in lipid parameters from baseline to week 8 by atorvastatin dose assignment in children and adolescents with heterozygous familial hypercholesterolemia

\begin{tabular}{|c|c|c|c|c|c|c|}
\hline \multirow[t]{2}{*}{ Variable } & \multicolumn{3}{|l|}{ Tanner stage 1} & \multicolumn{3}{|l|}{ Tanner stage $\geq 2$} \\
\hline & $\begin{array}{l}\text { Patients who } \\
\text { stayed at } 5 \mathrm{mg} \\
(n=5)\end{array}$ & $\begin{array}{l}\text { Patients who } \\
\text { increased to } \\
10 \mathrm{mg}(n=10)\end{array}$ & $\begin{array}{l}\text { All patients } \\
(n=15)\end{array}$ & $\begin{array}{l}\text { Patients who } \\
\text { stayed at } 10 \mathrm{mg} \\
(n=9)\end{array}$ & $\begin{array}{l}\text { Patients who } \\
\text { increased to } 20 \mathrm{mg} \\
(n=15)\end{array}$ & $\begin{array}{l}\text { All patients } \\
(n=24)\end{array}$ \\
\hline \multicolumn{7}{|c|}{ Low density lipoprotein cholesterol $(\mathrm{mmol} / \mathrm{l})$} \\
\hline Baseline mean & $4.87 \pm 0.48$ & $6.37 \pm 1.10$ & $5.87 \pm 1.18$ & $5.11 \pm 0.65$ & $6.23 \pm 1.00$ & $5.81 \pm 1.03$ \\
\hline End point mean & $3.06 \pm 0.54$ & $3.66 \pm 0.80$ & $3.46 \pm 0.76$ & $3.12 \pm 0.40$ & $3.63 \pm 0.43$ & $3.44 \pm 0.48$ \\
\hline$\%$ Change from baseline & $-36.78 \pm 11.16$ & $-42.70 \pm 6.45$ & $-40.72 \pm 8.41$ & $-38.45 \pm 7.84$ & $-40.39 \pm 11.71$ & $-39.66 \pm 10.28$ \\
\hline \multicolumn{7}{|l|}{ Total cholesterol $(\mathrm{mmol} / \mathrm{l})$} \\
\hline Baseline mean & $6.76 \pm 0.46$ & $8.58 \pm 1.06$ & $7.97 \pm 1.25$ & $6.92 \pm 0.71$ & $8.40 \pm 1.10$ & $7.84 \pm 1.20$ \\
\hline End point mean & $4.87 \pm 0.39$ & $5.39 \pm 0.75$ & $5.21 \pm 0.69$ & $4.66 \pm 0.65$ & $5.18 \pm 0.49$ & $4.99 \pm 0.60$ \\
\hline$\%$ Change from baseline & $-27.80 \pm 5.56$ & $-37.17 \pm 5.28$ & $-34.05 \pm 6.90$ & $-32.43 \pm 8.53$ & $-37.45 \pm 9.89$ & $-35.57 \pm 9.54$ \\
\hline \multicolumn{7}{|l|}{ Triglycerides (mmol/l) } \\
\hline Baseline mean & $0.76 \pm 0.15$ & $0.95 \pm 0.27$ & $0.89 \pm 0.25$ & $1.03 \pm 0.37$ & $1.20 \pm 0.50$ & $1.14 \pm 0.46$ \\
\hline End point mean, $\mathrm{mmol} / \mathrm{L}$ & $0.79 \pm 0.34$ & $0.79 \pm 0.23$ & $0.79 \pm 0.26$ & $0.72 \pm 0.24$ & $0.92 \pm 0.42$ & $0.84 \pm 0.37$ \\
\hline$\%$ Change from baseline & $1.69 \pm 31.48$ & $-9.88 \pm 33.31$ & $-6.02 \pm 32.06$ & $-20.94 \pm 39.24$ & $-21.11 \pm 23.85$ & $-21.05 \pm 29.69$ \\
\hline \multicolumn{7}{|c|}{ High density lipoprotein cholesterol (mmol/l) } \\
\hline Baseline mean & $1.35 \pm 0.12$ & $1.45 \pm 0.29$ & $1.41 \pm 0.25$ & $1.17 \pm 0.18$ & $1.18 \pm 0.23$ & $1.18 \pm 0.21$ \\
\hline End point mean & $1.39 \pm 0.31$ & $1.38 \pm 0.21$ & $1.38 \pm 0.24$ & $1.24 \pm 0.34$ & $1.11 \pm 0.23$ & $1.16 \pm 0.28$ \\
\hline$\%$ Change from baseline & $2.50 \pm 15.02$ & $-2.84 \pm 14.49$ & $-1.06 \pm 14.36$ & $5.99 \pm 21.02$ & $-5.19 \pm 17.76$ & $-1.00 \pm 19.40$ \\
\hline \multicolumn{7}{|c|}{ Very low density lipoprotein cholesterol ( $\mathrm{mmol} / \mathrm{l})$} \\
\hline Baseline mean & $0.54 \pm 0.15$ & $0.76 \pm 0.30$ & $0.69 \pm 0.27$ & $0.65 \pm 0.11$ & $0.99 \pm 0.31$ & $0.86 \pm 0.30$ \\
\hline End point mean & $0.42 \pm 0.20$ & $0.35 \pm 0.15$ & $0.37 \pm 0.16$ & $0.30 \pm 0.12$ & $0.44 \pm 0.21$ & $0.39 \pm 0.19$ \\
\hline$\%$ Change from baseline & $-12.31 \pm 57.38$ & $-50.29 \pm 21.44$ & $-37.63 \pm 39.75$ & $-53.61 \pm 19.21$ & $-52.38 \pm 29.34$ & $-52.84 \pm 25.55$ \\
\hline \multicolumn{7}{|l|}{ Apolipoprotein A-I (g/l) } \\
\hline Baseline mean & $1.42 \pm 0.20$ & $1.45 \pm 0.20$ & $1.44 \pm 0.19$ & $1.29 \pm 0.20$ & $1.24 \pm 0.15$ & $1.26 \pm 0.17$ \\
\hline End point mean & $1.40 \pm 0.14$ & $1.40 \pm 0.20$ & $1.40 \pm 0.18$ & $1.24 \pm 0.25$ & $1.17 \pm 0.22$ & $1.20 \pm 0.23$ \\
\hline$\%$ Change from baseline & $-1.24 \pm 8.49$ & $-3.37 \pm 9.35$ & $-2.66 \pm 8.82$ & $-2.60 \pm 20.22$ & $-4.82 \pm 16.13$ & $-3.98 \pm 17.37$ \\
\hline \multicolumn{7}{|l|}{ Apolipoprotein B (g/l) } \\
\hline Baseline mean & $1.09 \pm 0.13$ & $1.49 \pm 0.24$ & $1.35 \pm 0.28$ & $1.26 \pm 0.14$ & $1.52 \pm 0.18$ & $1.42 \pm 0.21$ \\
\hline End point mean & $0.79 \pm 0.12$ & $0.90 \pm 0.15$ & $0.86 \pm 0.15$ & $0.85 \pm 0.10$ & $1.04 \pm 0.26$ & $0.97 \pm 0.23$ \\
\hline$\%$ Change from baseline & $-27.39 \pm 5.97$ & $-39.59 \pm 5.83$ & $-35.52 \pm 8.22$ & $-31.94 \pm 11.64$ & $-31.26 \pm 18.57$ & $-31.51 \pm 16.04$ \\
\hline \multicolumn{7}{|c|}{ Low density lipoprotein cholesterol/high-density lipoprotein-cholesterol } \\
\hline Baseline mean & $3.63 \pm 0.39$ & $4.63 \pm 1.69$ & $4.30 \pm 1.46$ & $4.46 \pm 0.79$ & $5.41 \pm 1.11$ & $5.05 \pm 1.09$ \\
\hline End point mean & $2.33 \pm 0.72$ & $2.70 \pm 0.77$ & $2.58 \pm 0.75$ & $2.63 \pm 0.56$ & $3.44 \pm 0.87$ & $3.13 \pm 0.85$ \\
\hline$\%$ Change from baseline & $-36.49 \pm 16.48$ & $-39.95 \pm 11.02$ & $-38.80 \pm 12.59$ & $-40.82 \pm 8.88$ & $-35.84 \pm 12.54$ & $-37.71 \pm 11.37$ \\
\hline
\end{tabular}

was doubled for the remainder of the study) were at a much higher baseline LDL-C level regardless of Tanner stage cohort. The reductions in mean plasma levels of LDL-C, TC, and TG tended to be greater in the subjects whose atorvastatin dose was doubled at week 4 than in the subjects who continued to receive their original dose.

More variable results were collected for the other lipid parameters regarding atorvastatin dose level and cohort. For the TS $\geq 2$ subjects, the reductions in VLDL-C and Apo B were similar in the two dose groups. However, for the TS 1 subjects, the decrease in mean plasma VLDL-C $(-50.3 \%$ vs. $-12.3 \%)$ and Apo B $(-39.6 \%$ vs. $-27.4 \%)$ levels were greater among those whose dose had been doubled after the week 4 visit than among those who continued to receive their initial dose. For both cohorts, the decrease in Apo A-1 was somewhat larger among those whose dose had been doubled after the week 4 visit than among those who continued to receive their initial dose $(-3.4 \%$ vs. $-1.2 \%$ for TS $1 ;-4.8 \%$ vs. $-2.6 \%$ for TS $\geq 2$ ). The HDL-C levels were slightly improved in the patients who continued to receive their starting dose but slightly decreased for those whose dosage was doubled.

Temporal analysis of lipid parameters showed that the mean values for LDL-C and TC decreased by week 2 
among all TS 1 and TS $\geq 2$ subjects, whereas TG and HDL$\mathrm{C}$ varied considerably from week to week (Fig. 1). Plasma LDL-C and TC levels continued to decrease over the 8 -week study period for the patients whose dose had been doubled but exhibited maximum reductions by week 4 for those who remained at their initial dose (Fig. 2).

Flow-mediated dilation data were provided for 11 of 15 TS 1 subjects and for 17 of $24 \mathrm{TS} \geq 2$ subjects. For the TS 1 cohort, FMD values varied widely, ranging from $0.0 \%$ to $11.5 \%$ (mean, $6.3 \%$ ) at baseline and from $0.0 \%$ to $12.0 \%$ (mean, 5.5\%) at week 8. Similar wide variations in FMD values were observed for the TS $\geq 2$ cohort, ranging from $0.0 \%$ to $10.0 \%$ (mean, $3.9 \%$ ) at baseline and from $2.9 \%$ to $9.3 \%$ (mean, $4.9 \%$ ) at week 8 .

\section{Safety}

The safety results and the most commonly reported AEs for the two cohorts are shown in Table 3. No deaths, serious AEs, or premature discontinuations were reported. Overall, no difference in safety or tolerability was observed between the younger and older cohorts. A total of 16 AEs were reported from 9 patients in the TS 1 cohort (4 of which were considered treatment related) and 17 AEs from 13 patients in the TS $\geq 2$ cohort ( 2 of which were considered treatment related). The treatment-emergent AEs of abdominal pain, nausea, vomiting, and headache were experienced by two TS 1 patients, and treatment-emergent AEs of increased plasma ALT levels were experienced by two TS $\geq 2$ patients. A moderate but transient increase in blood creatinine, observed in a 9-year-old girl, was attributed to reduced water intake.

All treatment-emergent AEs were mild to moderate in intensity. The two episodes of increased plasma ALT levels occurred in two 13-year-old girls. One girl returned to "normal" levels during the study period. The other girl had a slightly elevated ALT level at the final visit. None of the ALT values at any of her other visits were elevated. No vital sign or ECG readings were documented as AEs.

\section{Discussion}

The pediatric patients with HeFH who participated in this study presented with very high LDL-C and TG levels and an HDL-C level within the normal range. The observed reductions in lipid values associated with a pediatric formulation of atorvastatin in the prepubertal TS 1 patients in this study were consistent with those seen in the TS $\geq 2$ patients and with reductions previously reported for other older pediatric populations $[1,7,14,16,19,21]$.

Overall, the mean values for LDL-C, TC, VLDL-C, and Apo B had decreased to a clinically significant extent by
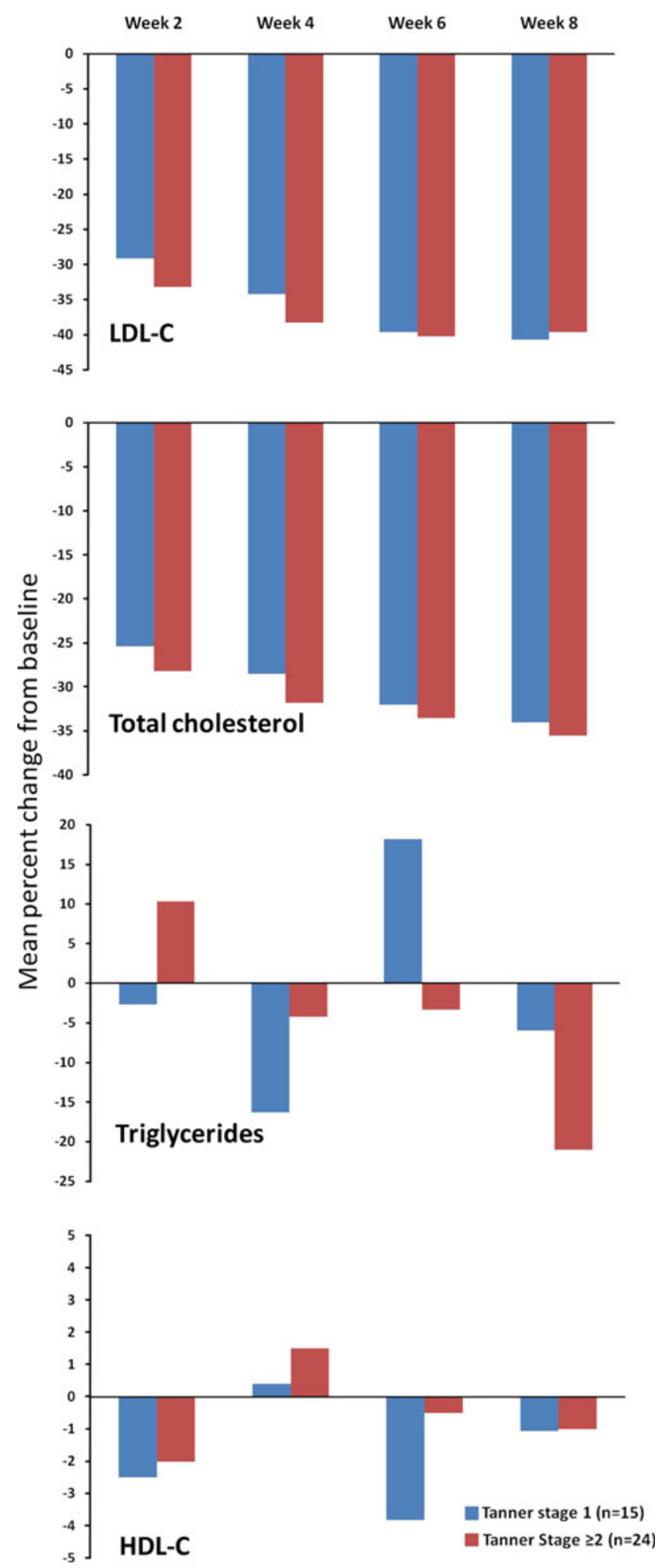

Fig. 1 Mean percentage change from baseline in lipid parameters of children and adolescents with heterozygous familial hypercholesterolemia who received daily atorvastatin 5 to $10 \mathrm{mg}$ Tanner stage (TS) 1 patients or 10 to $20 \mathrm{mg}$ (TS $\geq 2$ patients) for 8 weeks. TS 1 patients were treated initially with atorvastatin $5 \mathrm{mg} /$ day and $\mathrm{TS} \geq 2$ patients with $10 \mathrm{mg} /$ day. Doses were doubled at week 4 if the low density lipoprotein cholesterol (LDL-C) target concentration $(<130 \mathrm{mg} / \mathrm{dl}$ [3.35 mmol/l]) was not achieved 
Fig. 2 Temporal change from baseline reductions in lowdensity lipoprotein cholesterol (LDL-C) (top) and total cholesterol (TC) (bottom) of Tanner stage (TS) 1 and TS $\geq 2$ patients with heterozygous familial hypercholesterolemia stratified by a atorvastatin dose increase at week 4

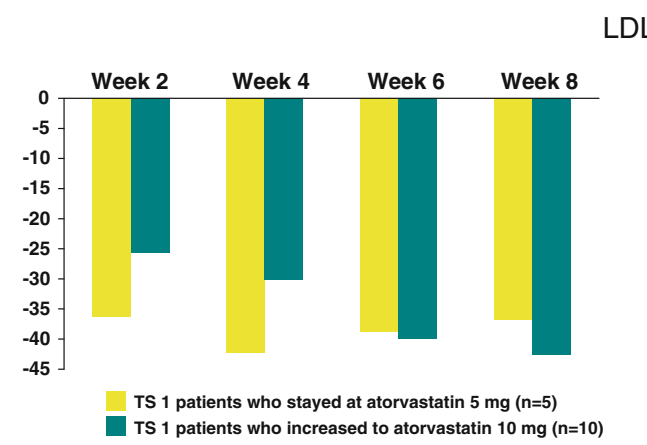

LDL-C

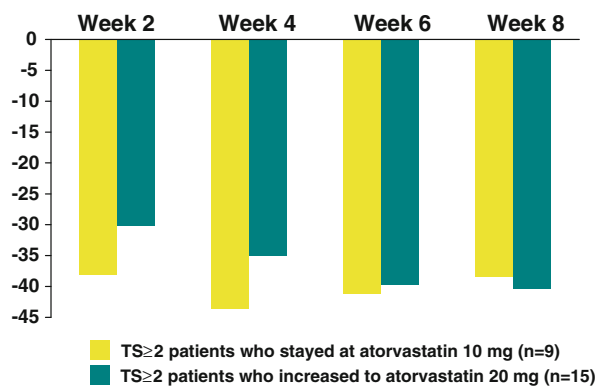

TC
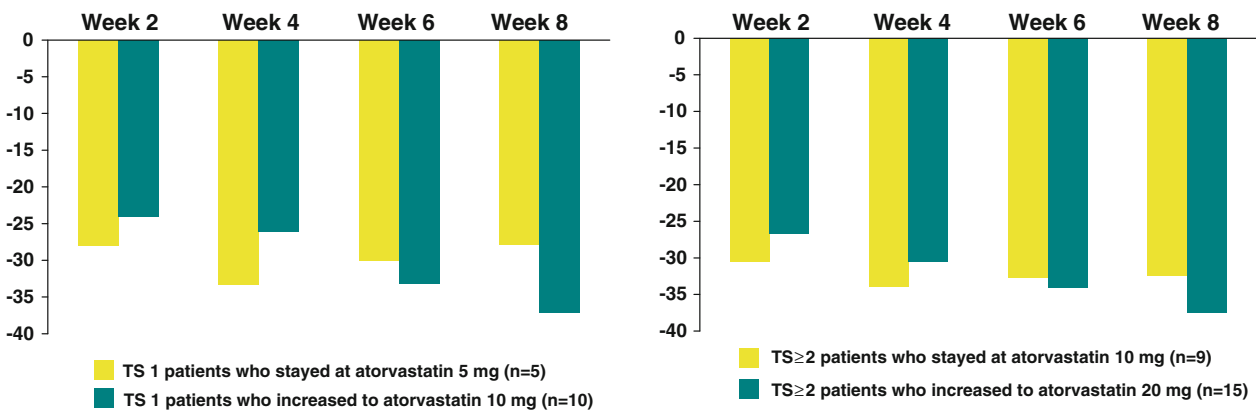

week 2 among all TS 1 and TS $\geq 2$ patients. The patients in each cohort whose dose was doubled had generally higher mean baseline lipid values than those not requiring a dose increase. For these subjects, additional improvements in lipid profile were observed as early as 2 weeks after the dose escalation. The mean percentage decreases in lipid parameters generally were similar for the two Tanner stage cohorts regardless whether the subjects remained at their initial dose or received a doubled dose. Furthermore, atorvastatin (5-10 $\mathrm{mg}$ for the TS 1 patients and 10-20 mg for the TS $\geq 2$ patients) was well tolerated in this study, and no safety issues were identified over the 8-week study duration. No clinically significant difference in tolerability or safety was observed between the younger and older cohorts.

Previous evidence has demonstrated the efficacy and safety of atorvastatin for pediatric patients. In a doubleblind, placebo-controlled clinical trial involving 187 children 10 to 17 years of age at TS $\geq 2$ with known familial hypercholesterolemia or severe hypercholesterolemia, the administration of atorvastatin (10-20 mg/day) for 12 months proved to be effective and well tolerated for the treatment of elevated lipid levels in both the children and the adolescents [21]. At week 26, atorvastatin 10 to $20 \mathrm{mg}$ compared with placebo was associated with significant reductions in LDL-C ( $-40 \%$ vs. $-0.4 \%)$, TC ( $-32 \%$ vs. $-1.5 \%)$, TG ( $-12 \%$ vs. $+1.0 \%)$, and Apo B ( $-34 \%$ vs. $+0.7 \%)$. Also HDL-C increased with atorvastatin compared with placebo $(+2.8 \%$ vs. $-1.8 \%)$.
In a compassionate use study, 46 children (11 age $<10$ years and 35 ages 11-17 years) with severe dyslipidemia, including 30 with homozygous $\mathrm{FH}$, atorvastatin was well-tolerated up to 3 years at titrated doses across the full available 10- to 80-mg dose range (data on file). Although the modification in lipid levels after the treatment of prepubertal TS 1 patients with atorvastatin observed in this study was not markedly different from that previously observed in other older pediatric populations $[1,7,14,16$, $19,21]$, several methodologic aspects of our study merit discussion. One limitation of the study was the necessarily small sample size. Although the sample small size was small, all the patients completed the study, treatment compliance was high. In addition, no data points were missing. Therefore, no imputations were necessary. Furthermore, the study was an open-label uncontrolled study that included only patients with FH.

However, as described earlier, in keeping with the need to limit the exposure of younger children, the design of this 8-week pharmacokinetic and pharmacodynamic study was thoroughly discussed with PDCO and endorsed by it. This study used well-established and validated modern population pharmacokinetic modeling approaches $[9,11]$, taking the minimal blood samples required from each child while obtaining robust population pharmacokinetic data.

Although the study design and small sample size precludes any definitive statements, overall, the PK of atorvastatin in this subgroup of pediatric patients does not appear to differ markedly from that in adults. The most 
Table 3 Incidence of all-cause TEAEs (and treatment-related AEs) reported in patients during 8 weeks of atorvastatin treatment

\begin{tabular}{|c|c|c|}
\hline \multirow[t]{2}{*}{ AEs } & \multicolumn{2}{|c|}{ No. evaluable patients } \\
\hline & $\begin{array}{l}\text { Tanner stage } 1 \\
(n=15)\end{array}$ & $\begin{array}{l}\text { Tanner stage } \geq 2 \\
(n=24)\end{array}$ \\
\hline All & $9(2)$ & $13(2)$ \\
\hline Serious TEAEs & 0 & 0 \\
\hline Severe or very severe TEAEs & 0 & 0 \\
\hline Discontinued due to a TEAE & 0 & 0 \\
\hline \multicolumn{3}{|l|}{ MedDRA preferred term } \\
\hline Nasopharyngitis & 1 & 2 \\
\hline $\begin{array}{l}\text { Viral upper respiratory } \\
\text { tract infection }\end{array}$ & 3 & 0 \\
\hline Headache & $2(1)$ & 1 \\
\hline Gastroenteritis & 1 & 1 \\
\hline $\begin{array}{l}\text { Alanine aminotransferase } \\
\text { increased }\end{array}$ & 0 & $2(2)$ \\
\hline Abdominal pain & $1(1)$ & 0 \\
\hline Nausea & $1(1)$ & 0 \\
\hline Toothache & 0 & 1 \\
\hline Vomiting & $1(1)$ & 0 \\
\hline Pain & 0 & 1 \\
\hline Bronchopneumonia & 1 & 0 \\
\hline Ear infection & 0 & 1 \\
\hline Gastritis viral & 1 & 0 \\
\hline Influenza & 0 & 1 \\
\hline $\begin{array}{l}\text { Lower respiratory tract } \\
\text { bacterial infection }\end{array}$ & 0 & 1 \\
\hline Tonsillitis & 0 & 1 \\
\hline Viral rhinitis & 1 & 0 \\
\hline Hand fracture & 0 & 1 \\
\hline Blood creatinine increased & 1 & 0 \\
\hline Arthralgia & 0 & 1 \\
\hline Musculoskeletal pain & 0 & 1 \\
\hline Pain in extremity & 0 & 1 \\
\hline Asthma & 1 & 0 \\
\hline Rhinitis allergic & 0 & 1 \\
\hline Urticaria & 1 & 0 \\
\hline
\end{tabular}

$A E$ adverse event; MedDRA Medical Dictionary for Regulatory Activities, version 12.0; TEAEs treatment emergent adverse events

important finding in the population PK analysis was that atorvastatin clearance $(\mathrm{CL} / \mathrm{F})$ in pediatric subjects appeared similar to that in adults when scaled by subject weight. Body weight also was the only covariate influencing atorvastatin $\mathrm{CL} / \mathrm{F}$ [12]. Other covariates such as age, gender, and Tanner stage had little influence on the PK parameters tested. At week 8 , on the average, the percentage change from baseline was approximately $40 \%$ for LDL-C and $30 \%$ for TC over the range of atorvastatin and o-hydroxyatorvastatin exposures [12].
Overall, there appeared to be no evidence of variations in lipid effects between Tanner stage patients or across the range of atorvastatin and o-hydroxyatorvastatin exposures. This likely is due to the Tanner stage-dependent doses and the titration dosing design in this study, in which the patients were evaluated for dose escalation based on target levels or reduction in LDL-C.

Approximately two-thirds of both Tanner stage cohorts (10 of $15 \mathrm{TS} 1$ patients and 15 of $24 \mathrm{TS} \geq 2$ patients) in this study had a much higher LDL-C at baseline and required up-titration at week 4 . For these patients, doubling the dose to 10 or $20 \mathrm{mg}$ (TS 1 and TS $>2$ cohorts, respectively) provided additional LDL-C reduction. Approximately 50\% of the patients reached the target $130 \mathrm{mg} / \mathrm{dl}(3.35 \mathrm{mmol} / \mathrm{l})$ at week 8. Currently, the evidence is limited regarding what target LDL-C level should be attained [5, 15]. Both the American Heart Association [17] and the American Academy of Pediatrics (AAP) [4] have recommended that LDL-C target levels as low as $130 \mathrm{mg} / \mathrm{dl}(3.35 \mathrm{mmol} / \mathrm{l})$ should be set for $\mathrm{HeFH}$ children. In clinical practice, a prescribing physician may consider titrating to a higher atorvastatin dose than those used in this study (i.e., above $10 \mathrm{mg} /$ day for TS 1 and above $20 \mathrm{mg} /$ day for TS $\geq 2$ ) to achieve the LDL-C target, taking into account patient risk and benefit profiles.

Use of plasma lipid levels as a reliable surrogate marker for coronary heart disease is well established for adults, and the main target of lipid-lowering management depends on the patient's level of risk. Recent evidence of statin use in children 8 to 17 years of age also indicates that a moderate reduction in LDL-C of $25 \%$ to $30 \%$ over a 2 -year period significantly decreases the rate of carotid artery intima thickening, a parameter often used as a predictor of coronary risk in adults [21].

To provide additional exploratory data showing the effects of atorvastatin on endothelial function in this population, a separate analysis of FMD was undertaken. Previous studies have shown significant improvement in endothelial dysfunction toward normal levels after short-term statin therapy in children with FH [20]. The current substudy showed a wide range of observed individual FMD values at baseline and at week 8 for both cohorts, but no meaningful conclusions could be drawn. Finally, the short time frame of this study precluded safety assessments of atorvastatin in TS 1 children with respect to growth and development.

In conclusion, clinically meaningful reductions in LDLC, TC, VLDL-C, and Apo B were observed with atorvastatin in TS 1 and TS $\geq 2$ patients with $\mathrm{HeFH}$. Atorvastatin also was well tolerated in this population, and no patients withdrew prematurely from this 8-week study. However, the effect that long-term use of statins has on the TS 1 patient is not established, and treatment should be administered under close pediatric supervision. 
Acknowledgments This study was sponsored by Pfizer Inc. Editorial support was provided by Malcolm Darkes and John Bilbruck at UBC Scientific Solutions Ltd and funded by Pfizer Inc. The authors acknowledge Mary-Ellen Boers, Dave Windsor, Michael Mitnick, Luz Angela Cubillos (all full-time employees of Pfizer), Euridiki Drogari (University of Athens, Greece), Claude Gagne (Centre Hospitalier de I'Universite Laval, Quebec, Canada), and Gisle Langslett (Oslo University Hospital, Norway) for their contributions to the study.

Open Access This article is distributed under the terms of the Creative Commons Attribution Noncommercial License which permits any noncommercial use, distribution, and reproduction in any medium, provided the original author(s) and source are credited.

\section{References}

1. Clauss SB, Holmes KW, Hopkins P et al (2005) Efficacy and safety of lovastatin therapy in adolescent girls with heterozygous familial hypercholesterolemia. Pediatrics 116:682-688

2. Corretti MC, Anderson TJ, Benjamin EJ et al (2002) Guidelines for the ultrasound assessment of endothelial-dependent flowmediated vasodilation of the brachial artery: a report of the International Brachial Artery Reactivity Task Force. J Am Coll Cardiol 39:257-265

3. Cuthbert JA, East CA, Bilheimer DW et al (1986) Detection of familial hypercholesterolemia by assaying functional low-density-lipoprotein receptors on lymphocytes. N Engl J Med 314:879-883

4. Daniels SR, Greer FR, Committee on Nutrition (2008) Lipid screening and cardiovascular health in childhood. Pediatrics 122:198-208

5. de Ferranti S, Ludwig DS (2008) Storm over statins: the controversy surrounding pharmacologic treatment of children. N Engl J Med 359:1309-1312

6. de Jongh S, Lilien MR, op't Roodt J et al (2002) Early statin therapy restores endothelial function in children with familial hypercholesterolemia. J Am Coll Cardiol 40:2117-2121

7. de Jongh S, Ose L, Szamosi T et al (2002) Efficacy and safety of statin therapy in children with familial hypercholesterolemia: a randomized, double-blind, placebo-controlled trial with simvastatin. Circulation 106:2231-2237

8. European Medicines Agency (2006) Assessment of the paediatric needs for cardiovascular products. http://www.emea.europa.eu/pdfs/ human/paediatrics/43964906en.pdf. Retrieved 14 June 2010

9. European Medicines Agency (2008) Guideline on reporting the results of population pharmacokinetic analyses. http://www.ema.eur opa.eu/pdfs/human/ewp/18599006enfin.pdf. Retrieved 14 June 2010

10. European Medicines Agency (2008) Meeting highlights from the Paediatric Committee, 2-4 June 2008. www.ema.europa.eu/ pdfs/human/pdco/29649408en.pdf. Retrieved 23 June 2010

11. Food and Drug Administration (1999) Guidance for industry: population pharmacokinetics. http://www.fda.gov/downloads/Drugs/ GuidanceComplianceRegulatoryInformation/Guidances/ucm072 137.pdf. Retrieved 18 June 2010

12. Gandelman K, Glue P, Malhotra B, Gastonguay M, Knebel W, Jen F (2010) Population pharmacokinetic and pharmacodynamic modeling of atorvastatin and its active metabolite in pediatric patients with heterozygous familial hypercholesterolemia. Clin Pharmacol Ther 87:S61-S62

13. Goldstein JL, Brown MS (1992) Familial hypercholesterolemia. In: Stanburg JB, Wyngaarden JB, Fredrickson DS (eds) The Metabolic basis of inherited disease, 7th edn. McGraw-Hill, New York, pp 1215-1250

14. Knipscheer HC, Boelen CC, Kastelein JJ et al (1996) Short-term efficacy and safety of pravastatin in 72 children with familial hypercholesterolemia. Pediatr Res 39:867-871

15. Lebenthal Y, Horvath A, Dziechciarz P et al (2010) Are treatment targets for hypercholesterolemia evidence based? Systematic review and meta-analysis of randomised controlled trials. Arch Dis Child 95:673-680

16. McCrindle BW, Ose L, Marais AD et al (2003) Efficacy and safety of atorvastatin in children and adolescents with familial hypercholesterolemia or severe hyperlipidemia: a multicenter, randomized, placebo-controlled trial. J Pediatr 143:74-80

17. McCrindle BW, Urbina EM, Dennison BA et al (2007) Drug therapy of high-risk lipid abnormalities in children and adolescents: a scientific statement from the American Heart Association Atherosclerosis, Hypertension, and Obesity in Youth Committee, Council of Cardiovascular Disease in the Young, with the Council on Cardiovascular Nursing. Circulation 115:1948-1967

18. Rodenburg J, Vissers MN et al (2007) Statin treatment in children with familial hypercholesterolemia: the younger, the better. Circulation 116:664-668

19. Stein EA, Illingworth DR, Kwiterovich PO Jr et al (1999) Efficacy and safety of lovastatin in adolescent males with heterozygous familial hypercholesterolemia: a randomized controlled trial. JAMA 281:137-144

20. Vuorio AF, Aalto-Setälä K, Koivisto UM et al (2001) Familial hypercholesterolaemia in Finland: common, rare, and mild mutations of the LDL receptor and their clinical consequences. Finnish FH Group. Ann Med 33:410-421

21. Wiegman A, Hutten BA, de Groot E et al (2004) Efficacy and safety of statin therapy in children with familial hypercholesterolemia: a randomized controlled trial. JAMA 292:331-337 